\section{AB1301 CARDIOVASCULAR RISK AGE AND VASCULAR AGE ESTIMATIONS IN PREDICTING CARDIOVASCULAR EVENTS IN RHEUMATOID ARTHRITIS PATIENTS}

G. Wibetoe $^{1}$, J. Sexton ${ }^{1}$, E. Ikdahl ${ }^{1}$, S. Rollefstad ${ }^{1}$, G. Kitas ${ }^{2,3}$, P. van Riel ${ }^{4}$, S. Gabriel ${ }^{5}$, T.K. Kvien ${ }^{1}$, K. Douglas ${ }^{3}$, A. Sandoo ${ }^{3}$, E.E. Arts ${ }^{6}$, S. WållbergJonsson $^{7}$, S. Rantapää Dahlqvist ${ }^{7}$, G. Karpouzas ${ }^{8}$, P.H. Dessein ${ }^{9,10}$, L. Tsang ${ }^{11}$ H. El-Gabalawy ${ }^{12}$, C.A. Hitchon ${ }^{12}$, V. Pascual-Ramos ${ }^{13}$, I. Contreas-Yaňes ${ }^{13}$, P. P. Stikakis ${ }^{14}$, M.A. González-Gay ${ }^{15}$, I.J. Colunga-Pedraz ${ }^{16}$, D.A. GalarzaDelgado $^{17}$, J.R. Azpiri-Lopez ${ }^{18}$, C.S. Crowson ${ }^{19}$, A.G. Semb ${ }^{1} .{ }^{1}$ DIAKONHJEMMET HOSPITAL, Oslo, Norway; ${ }^{2}$ School of Sport, Exercise and Rehabilitation, University of Birmingham, Birmingham; ${ }^{3}$ Dudley Group NHS Foundation Trust, West Midlands, UK; ${ }^{4}$ Radboud university medical center, Radboud Institute for Health Sciences, IQ healthcare, Nijmegen, Netherlands; ${ }^{5}$ Rutgers Robert Wood Johnson Medical School, New Brunswick, NJ, USA; ${ }^{6}$ Department of Rheumatic Diseases, Radboud University Nijmegen Medical Centre, Nijmegen, Netherlands; ${ }^{7}$ Department of Public Health and Clinical Medicine, Rheumatology, Umeå University, Umeå, Sweden; ${ }^{8}$ Division of Rheumatology, Harbor-UCLA Medical Center, Torrance, CA, USA; ${ }^{9}$ Vrije Universiteit Brussel; ${ }^{10}$ Universitair Ziekenhuis Brussel; ${ }^{11}$ Rheumatology, Universitair Ziekenhuis Brussel, Brussel, Belgium; ${ }^{12}$ University of Manitoba, Winnipeg, MB, Canada; ${ }^{13}$ Instituto Nactional de Ciencias Médicas y Nutrición Salvador Zubirán, México City, Mexico; ${ }^{14}$ First Department of Propedeutic Internal Medicine, National and Kapodistrian University of Athens, Athens, Greece; ${ }^{15}$ Rheumatology, Hospital Universitario Marqués de Valdecilla, IDIVAL, Santander, Universidad de Cantabria, Spain, Santander, Spain; ${ }^{16} 18$ Rheumatology, Hospital Universitario, UANL, Monterrey; ${ }^{17}$ Hospital Universitario, UANL; ${ }^{18}$ Cardiology, Hospital Universitario, UANL, Monterrey, Mexico;

${ }^{19}$ Department of Medicine, Division of Rheumatology, Mayo Clinic, Rochester, MN, USA

Background: Rheumatoid arthritis (RA) patients are at high risk of cardiovascular disease (CVD). Risk age estimations are recommended as adjuncts to assessment of absolute 10 year risk of fatal CVD events. Two risk age models based on the Systematic Coronary Risk Evaluation (SCORE) algorithm have been developed; the cardiovascular risk age and the vascular age.

Objectives: We aimed to compare the discriminative ability of cardiovascular risk age and vascular age among RA patients and in subgroups of RA patients.

Methods: Patients with RA were included from an international consortium, aged 30-70 years at baseline. Those with prior CVD, diabetes and/or users of lipid-lowering and/or antihypertensive therapy at baseline were excluded. Cardiovascular risk age was estimated based on chronologic age, smoking status, total cholesterol and systolic blood pressure at baseline. Vascular age was derived from the 10 year risk of CVD according to the SCORE algorithm, with or without high density lipoprotein cholesterol, using the equations for low and high risk countries. Performance of each risk age model in predicting CVD events was assessed by Cstatistics.

Results: Among 1867 patients included, $74 \%$ were female, median (inter-quartile range) age and disease duration were $52.0(44.0,59.9)$ and $0.6(0.1,6.4)$ years, $72.5 \%$ were rheumatoid factor positive, $24.7 \%$ were using glucocorticoids and $10.3 \%$ were using biologics at baseline. Overall, 144 CVD events occurred. Median follow-up time was $5.0(2.6,9.3)$ years. C-indices across risk models ranged from 0.71 to 0.73 with standard errors of 0.03 . Across prediction models, the lowest observed concordance was found among women and in glucocorticoid users and in those with new-onset disease ( $\leq 1$ year). Additional analyses including RA patients on cardio preventive therapy yielded slightly lower c-indexes. Since SCORE was developed for use in Europe, we performed analyses on European RA patients, which yielded similar results. Certain characteristics were associated with low concordance, but standard errors were high (data not shown).

Conclusions: The cardiovascular risk age and vascular age models have comparable performance in predicting CVD in RA patients. The influence of RA disease characteristics on the predictive ability of these prediction models remains inconclusive.

Disclosure of Interest: None declared

DOI: 10.1136/annrheumdis-2018-eular.3699

\section{AB1302 RESULTS FROM THE PROSPECTIVE NATIONWIDE NORWEGIAN PREGNANCY QUALITYREGISTER REVNATUS}

H. Bjørngaard ${ }^{1}$, B. Jakobsen ${ }^{1}$, H.S.S. Koksvik ${ }^{1}$, M. Wallenius ${ }^{1,2} .{ }^{1}$ Norwegian $^{2}$ National Advisory Unit on Pregnancy and Rheumatic Diseases, RHEUMATOLOGY UNIT, ST OLAVS HOSPITAL; ${ }^{2}$ INB (Institute of neuro medicine and movement science), NTNU Norwegian university of science and technology, Trondheim, Norway

Background: To establish a register to determine disease activity, pregnancy surveillance and safety of medication during pregnancy and lactation, and monitoring this group as a quality register.
Methods: The Norwegian pregnancy register RevNatus is designed as a nationwide, web-based longitudinal observational cohort study with 17 participating centres. Pregnant patients or patients planning a pregnancy with confirmed diag nosis of inflammatory rheumatic disease are eligible to be enrolled. The women are preferred enrolled before conception and with registrations each trimester, 6 weeks, 6 and 12 months postpartum. At baseline diagnosis, sociodemographic parameters, disease activity, anti-rheumatic medication, obstetric history, comorbidities and antibody status are reported. The register has been approved by the Norwegian Data Inspectorate and is run by The Norwegian National Advisory Unit on Pregnancy and Rheumatic Diseases.

Results: From February 2016 - January 2018597 women were included in RevNatus, mean age at inclusion was 30,8 (17-44). Among these patients $4 \%$ had only completed primary school, $24 \%$ secondary education and $72 \%$ had completed a university education. At inclusion $4 \%$ were smoking (general population $11 \%$ ) and $6 \%$ used snuff (general population 12\%). At registration 6 weeks after delivery $3 \%$ were smoking and $2 \%$ used snuff. Alltogether 346 women were registered with a control 6 weeks after delivery. Of these, 31 women experienced spontaneous abortion and one therapeutic abortion. Among the remaining, $314 \mathrm{had}$ live born infants including 7 twin births and one triplet birth. Among the women with registration 6 week postpartum, $260(75 \%)$ women were diagnosed with chronic inflammatory arthritis including rheumatoid arthritis, spondylarthritis, juvenile idiopathic arthritis and unspecified arthritis. Correspondingly, $79(23 \%)$ women were registered with inflammatory connective tissue disease (SLE, MCTD, poly-dermato myositis, systemic sclerosis), 3 with vasculitic disease (Takayasu's arteritis, Mb Behcet), and 5 with primary anti-phospholipid antibody (APS) syndrome. Mean disease duration (SD) for all diagnoses was 6,5 years. ${ }^{6,8}$ Corresponding, mean disease duration in women with RA was $6,7(4,1)$ years, in women with SLE 9,4 $(6,1)$ years and in women with JIA 20,7 (7,3) years. Mean gestational age at birth for all diagnoses was $38,7(2,5)$ weeks. The gestational age was lowest in SLE women 37,9 $(2,59)$ weeks. Mean gestational week for spontaneous abortion was at 11 weeks. Mean birthweight (SD) was $3268^{758,7}$ gram in offspring of women with RA and 3133 gram in women with SLE. Overall $71(22 \%)$ women had caesarean deliveries, $14 \%$ were acute and $9 \%$ planned caesarean deliveries. Six weeks postpartum 258 women $(82,7 \%)$ were breastfeeding their babies.

Conclusions: The Norwegian pregnancy register RevNatus was established to study the course and outcomes of pregnancies in women with inflammatory rheumatic diseases as well as increased knowledge on the use and safety of treatments during pregnancy and lactation. The results of the register gives data to monitor the quality of the treatment for this group of patients.

Disclosure of Interest: None declared

DOI: 10.1136/annrheumdis-2018-eular.4549

\section{AB1303 ANALYSIS OF SYMPTOMS IN VERY EARLY PHASE IN PATIENTS WITH ADULT-ONSET STILL'S DISEASE}

H. Tamai, Y. Kaneko, T. Takeuchi. Division of Rheumatology, Department of Internal Medicine, Keio University School of Medicine, Tokyo, Japan

Background: The initial symptoms of Adult-onset Still Disease (AOSD) are nonspecific and confusing with those of common cold, which can lead to the delay of diagnosis and treatment.

Objectives: To clarify characteristic features in AOSD in very early phase and to find the key symptoms and markers to an earlier diagnosis and treatment.

Methods: We retrospectively reviewed consecutive AOSD patients in our hospital from April 2012 to July 2017. Symptoms and laboratory data before treatment were collected form their charts and analysed.

Results: A total of 62 patients were enrolled. The mean age at diagnosis was 45.9 and female was $81 \%$. The duration from the first symptoms to the first visit to a medical facility was 18.7 days, from the first visit to the first blood test was 5.8 days, from the first blood test to the fulfilment of Yamaguchi's Criteria was 11.0 days, and from the fulfilment of Yamaguchi's Criteria to the treatment was 22.2 days. During the course of developing the disease, fever was found in all patients, skin lesion in $91.9 \%$, arthralgia in $87.1 \%$, sore throat in $66.1 \%$. Laboratory and imaging tests demonstrated liver enzyme elevation in $82.2 \%$, white blood cell count (WBC) increase in $80.6 \%$, lymphadenopathy/splenomegaly in $72.6 \%$, negative RF and ANA in $56.5 \%$, ferritin elevation in $82.3 \%$. Laboratory tests before treatment demonstrated that the mean WBC count 15272.3/uL, AST $101.8 \mathrm{U} / \mathrm{L}$, CRP $15.1 \mathrm{mg} / \mathrm{dl}$, and ferritin $10103.1 \mathrm{ng} / \mathrm{ml}$. Patients who presented with sore throat as the first symptom fulfilled Yamaguchi's Criteria in 13.6 days after the onset, which was shorter than patients with any other symptoms (fever, 24.0 days; arthralgia, 45.7 days; skin lesion, 31.5 days). Before treatment, patients with sore throat as the first symptom showed highest AST of $139.6 \mathrm{U} / \mathrm{L}$ (fever, $95.3 \mathrm{U} / \mathrm{L}$; arthralgia, $100.7 \mathrm{U} / \mathrm{L}$; skin lesion, $71.6 \mathrm{U} / \mathrm{L}$ ) and highest ferritin of $19791.8 \mathrm{ng} / \mathrm{m}$ (fever, $9508.2 \mathrm{ng} / \mathrm{ml}$; arthralgia, $13071.5 \mathrm{ng} / \mathrm{ml}$; skin lesion, $8438.1 \mathrm{ng} / \mathrm{ml}$ ). However, the duration from the Yamaguchi's Criteria fulfilment to treatment initiation in patients with sore throat as the first symptom was 38.5 days, which was no less 